\title{
2011 - ein Jahr für Jubiläen und junge Allergologen
}

D ieses ist die erste Ausgabe des Allergo Journal in einem besonderen Jahr für die Allergologie, liegen doch die beiden Publikationen zur spezifischen Immuntherapie (SIT) von John Freeman (Further observations on the treatment of hay fever by hypodermic inoculations of pollen vaccine. Lancet 1911; 178: 814-7) und Leonard Noon (Prophylactic inoculation against hay fever. Lancet 1911; 177: 1572-3) 2011 seit 100 Jahren vor. Seit ihrer Jugend hat die Hyposensibilisierung ,medizinische Hoffähigkeit" vor allem durch vielfältige Forschungsarbeiten erlangt, die durch sinnvolle Kooperation zwischen akademischen Disziplinen, der Industrie und den Zulassungsbehörden Wirksamkeit und Indikationsstellung festigten und das Verständnis des Wirkmechanismus ermöglichten. Zu-

„Seit ihrer Jugend hat die Hyposensibilisierung ,medizinische Hoffähigkeit' durch Forschungsarbeiten erlangt, die Wirksamkeit und Indikationsstellung festigten." gleich führten sie zu vielfältigen Entwicklungen: von neuen Applikationsformen über modifizierte Allergene bis hin zu Kombinationen mit Pharmaka. Als weiteres Jubiläum 2011 feiert die DGAKI in diesem Jahr ihr 60-jähriges Bestehen. Beiden Ereignissen wird das Allergo Journal im Sommer ein Schwerpunktheft widmen.

In dieser Ausgabe des Allergo Journal finden Sie ab Seite 14 ein Interview mit Karl-Christian Bergmann und Jörg Kleine-Tebbe, in dem es um wesentliche Fragen der Auswertung klinischer Studien zur SIT geht. Diese Fragen dürften in den kommenden Monaten und Jahren, wenn strengere Kriterien zur Zulassung von Extrakten für die SIT angelegt werden, besondere Bedeutung gewinnen.

Durch Berücksichtigung rekombinant hergestellter Proteine von Bienenund Wespengift konnte ein wichtiger Fortschritt in der Diagnostik und Indikationsstellung der SIT bei Insektengiftallergien erlangt werden - ein Aspekt, den wir im dritten Teil der Serie „Molekulare Allergologie" ab Seite 22 beleuchten.

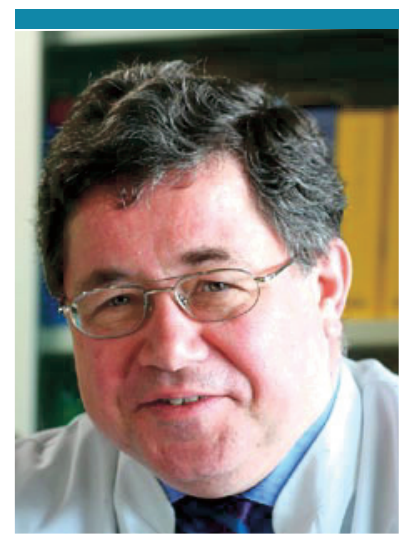

Prof. Dr. Hans F. Merk, Hautklinik der Medizinischen Fakultät, Universitätsklinikum der RWTH Aachen

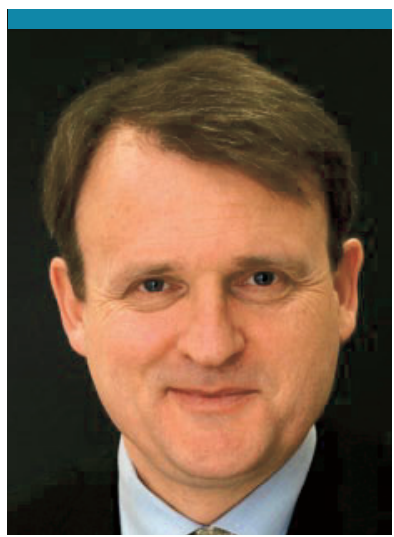

Prof. Dr. Thilo Jakob, Allergieabteilung \& Forschergruppe Allergologie, UniversitätsHautklinik Freiburg
Viele interessante Themen sind in den Abstracts des kommenden Mainzer Allergie-Workshops behandelt (Seite 25). Ein Höhepunkt der Tagung wird das Gründungstreffen der „Junior Members“" der DGAKI sein, auf dessen Verlauf wir alle gespannt sind.

Zentraler Kongress für alle Allergologen in Deutschland in diesem besonderen Jahr der Allergologie wird der 6 . Allergiekongress in Wiesbaden sein, der von Ludger Klimek und Wolfgang Schlenter organisiert wird. Wichtige

Hinweise zum Stand der Vorbereitungen finden Sie auf Seite 20. Auch das vollständige Pro„Ein Höhepunkt des Mainzer Allergie-Workshops wird das Gründungstreffen der ,Junior Members' der DGAKI sein." gramm, das eine spannende Tour durch alle aktuellen und interessanten Themen unseres Fachs bietet, wird bald zur Verfügung stehen.

Wir wünschen Ihnen eine anregende Lektüre und freuen uns darauf, das Jahr 2011 gemeinsam mit Ihnen zu erleben und zu gestalten.

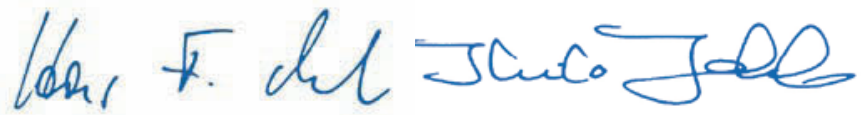

Prof. Dr. Hans F. Merk Prof. Dr. Thilo Jakob 